\title{
Les entours de l'œuvre. La littérature française contemporaine par elle-même
}

\author{
Mathilde Barraband \\ Université du Québec à Trois-Rivières \\ Jean-François Hamel \\ Université du Québec à Montréal
}

Un parergon vient contre, à côté et en plus de l'ergon, du travail fait, du fait, de l'œuvre mais il ne tombe pas à côté, il touche et coopère, depuis un certain dehors, audedans de l'opération.

Jacques Derrida, La Vérité en peinture 
«L'histoire de la littérature ne consiste pas seulement dans l'histoire des formes, mais aussi dans l'histoire des idées formulées et agissantes » (p. 76), écrivait Albert Thibaudet dans son Histoire de la littérature française. Le critique attitré de la Nouvelle Revue Française justifiait cet énoncé de méthode en soulignant l'influence de la pensée de Madame de Staël et du groupe de Coppet sur les écrivains des premières décennies du XIXe siècle. Plus récemment, Laurent Jenny prolongeait cette réflexion en rappelant, en ouverture d'un essai consacré à la figuration de la pensée dans les mouvements d'avant-garde, que «ce que nous appelons "littérature" ne se conçoit guère sans un corps d'idées, qui pour partie la constitue et pour partie l'interprète et lui donne sens » (p.7). Il va sans dire que ni Thibaudet ni Jenny ne rejetaient l'étude des particularités formelles des textes littéraires, pas plus qu'ils n'excluaient de l'histoire de la littérature les déterminations économiques et sociales qui circonscrivent à chaque époque l'espace des possibles. Ils entendaient plus modestement, plus banalement peut-être, rappeler que l'histoire de la littérature est aussi l'histoire de l'idée de littérature.

Loin de demeurer identique à elle-même, l'idée de littérature est l'objet de discussions passionnées, de débats houleux et, parfois, de polémiques violentes. N'ayant ni la rigueur du concept philosophique ni l'univocité de la proposition théorique, elle se manifeste à travers des images récurrentes et des réseaux métaphoriques; elle se déplace au gré des appropriations, variant non seulement d'une époque à l'autre, mais dans un même présent. Si l'idée de littérature s'exprime dans les œuvres, elle s'explicite souvent davantage dans leurs parerga et leurs entours, c'est-à-dire dans les lieux, parfois marginaux, toujours réflexifs, qui sont destinés à 
accueillir le commentaire des auteurs sur leur pratique et leur condition. À cet égard, le vaste ensemble de documents que Gérard Genette (1987) regroupait sous les catégories du péritexte et de l'épitexte constitue tout à la fois la fabrique et l'archive de l'idée de littérature. Les entretiens, les préfaces, les notes, les conférences, les hommages, les essais critiques, les journaux intimes et la correspondance disent en effet, souvent mieux que les œuvres elles-mêmes, en quoi celles-ci relèvent d'une certaine idée de la littérature et de son devenir.

L'espace littéraire français contemporain semble multiplier sans fin les lieux propres à mettre en œuvre cette réflexivité: les éditeurs, les médias électroniques, les institutions culturelles et les universités sollicitent constamment le retour des écrivains sur leur pratique ou celles de leurs pairs. Le succès des collections dédiées aux essais ou aux entretiens d'écrivains, le développement des études universitaires sur les écritures contemporaines, ou encore l'essor des blogs d'écrivains et des sites consacrés à la littérature actuelle ont considérablement élargi l'espace dévolu à la production du commentaire sur la littérature. Force est de constater que les écrivains français contemporains, qu'on disait peu enclins à adopter une posture de penseurs et d'historiens de la littérature, sont en fait nombreux à consacrer des travaux à leurs pairs, à expliciter la genèse de leurs projets d'écriture, à tenter de se situer dans le paysage littéraire et à débattre de l'idée de littérature. Tout se passe comme si la réception de la littérature dans l'espace public exigeait désormais, au même titre que l'art contemporain, le soutien de discours d'accompagnement qui en explicitent les raisons, la valeur et le sens. 
L'enjeu principal de ce dossier est de rendre compte des pratiques métalittéraires contemporaines, d'une part en identifiant les lieux où elles trouvent à se développer, d'autre part en tâchant de définir leurs visées et leurs fonctions. Efforts ou refus de définition de ce qu'est la littérature aujourd'hui, ces textes sont travaillés par la représentation, dans le double sens où l'écrivain s'y livre à une tentative de figuration de sa pratique et à la mise en scène de sa propre figure. Le dossier s'attache ainsi à faire apparaître les idées de littérature et les images de l'écrivain qui émanent de cette prolifération de textes «seconds». Qu'en est-il aujourd'hui des injonctions modernes et avant-gardistes des décennies précédentes? Que sont devenus les thèmes de la littérature comme instrument de connaissance, d'émancipation, de transgression, de transmission? Comment les entours de l'œuvre contribuent-ils à la construction de l'identité des écrivains dans la filiation ou le rejet de grandes figures du passé (écrivain engagé, intellectuel, clerc, lettré, voyant...)? Il s'agit d'interroger les événements, les idéologèmes qui servent aujourd'hui de socles identitaires, de marqueurs de reconnaissance, d'embrayeurs pour l'imaginaire. En somme, la question est celle-ci : si, de la plume des écrivains français d'aujourd'hui, il ne subsistait que les textes qui entourent le texte, les discours qui redoublent le discours, les parerga qui encadrent les œuvres, quel visage la littérature des trois dernières décennies donnerait-elle d'elle-même ${ }^{1}$ ?

\footnotetext{
1 Bien que plusieurs articles du dossier tendent à la remettre en question, nous adoptons ici la périodisation la plus largement admise, qui situe au tournant des années soixante-dix et quatre-vingt l'émergence de la littérature contemporaine en France. On pourra se reporter à ce sujet à la synthèse de D. Viart et B. Vercier (2008).
} 
En se penchant sur l'œuvre de Gérard Macé, largement sous le signe d'une écriture du commentaire, l'article de Laurent Demanze signale dès l'abord que l'écriture qu'on dit seconde n'est en rien subalterne, que l'entour de l'œuvre en constitue parfois le centre. Interrogeant les notes, scolies, manchettes, préfaces qui constituent les trames majeures du texte de Macé, il donne à voir la naissance d'une identité d'écrivain dans l'épreuve de l'altérité et interroge plus largement le geste de la référence érudite dans le contexte de la modernité. Audrey Camus propose quant à elle d'emboîter le pas de la fameuse marquise, qui sortit à cinq heures et occupa plusieurs générations d'écrivains détracteurs du genre romanesque. Le succès de la phrase attribuée à Paul Valéry, qui revient d'essais en fictions tout au long du Xxe siècle, n'a d'égal que le mystère qui entoure sa principale protagoniste, dont nul ne définit véritablement le visage littéraire. Il revient à Éric Chevillard d'avoir mis en scène cette marquise dans un bref récit publié en revue en 2002 et d'avoir ainsi achevé le travail de fictionnalisation du commentaire en germe chez ses prédécesseurs.

Comme le montre Jean-François Hamel, un tout autre récit, celui de l'Exode, obsède étrangement le discours des écrivains du second demi-siècle, lorsqu'ils s'attachent à définir une politique de la littérature. De Jean-Paul Sartre, Roland Barthes et Maurice Blanchot à Pierre Michon et Olivier Rolin, le récit de la sortie hors d'Égypte vers la Terre promise permet de nouer les « thèmes de l'oppression et de la liberté, de la loi et de la révolte, de l'ancien et du nouveau », qui furent ceux des avant-gardes $\mathrm{du} \quad \mathrm{xx}^{\mathrm{e}}$ siècle. Chez les deux écrivains contemporains, dont l'échange se noue d'article en hommage, l'Exode devient le signe de reconnaissance d'une génération 
littéraire née des paradoxes de Mai 68. C'est d'ailleurs au dialogue, souvent implicite et toujours contradictoire, que cette même génération continue d'entretenir avec Jean-Paul Sartre que Mathilde Barraband s'intéresse. Pierre Michon, Olivier Rolin, François Bon, Pierre Bergounioux, Didier Daeninckx, et Natacha Michel, tous publiés chez Verdier et passés au tournant des années 1980 de l'engagement militant à l'engagement dans la lettre, construisent au fil de leurs entretiens et essais une figure de l'écrivain à rebours de celle de l'écrivain engagé. C'est un autre héritage qu'Audrey Lasserre propose d'interroger: celui du féminisme chez les écrivaines françaises d'aujourd'hui. Analysant tous les entretiens d'écrivaines publiés dans Le Matricule des anges depuis son premier numéro, en 1992, elle y interroge l'absence de référence à la littérature et aux mouvements féministes. Sa réflexion est aussi l'occasion de questionner l'exercice de l'entretien et, plus particulièrement, la scénographie auctoriale qui s'y met en place par le biais des références invoquées mais aussi de l'énonciation.

Les articles de Lucie Bourassa et d'Alain Farah mettent en avant, si ce n'est un groupe, du moins un "réseau » littéraire, formé dans les années 1990 et qui renoue avec certaines pratiques des avant-gardes, notamment en s'appuyant sur les revues pour faire circuler leur pensée de la littérature et leurs fictions théoriques. Lucie Bourassa, tout en se concentrant sur les essais, péritextes et entretiens de Katalin Molnár, dont elle donne à lire et voir la création verbale, montre bien que, chez cette poétesse expérimentale, les élaborations d'une théorie du langage et d'une poétique ne sauraient être menées distinctement. De la même manière, chez Christophe Tarkos, le commentaire sur la littérature ne pouvait que mettre en œuvre ce qu'il préconise, et l'écriture sur y est toujours écriture. Alain 
Farah examine sa réponse à la revue Java, qui interrogeait des écrivains en 1997 sur la fin des avant-gardes. La série $\mathrm{d}^{\prime}$ ' " histoires » aux accents manifestaires que clame haut et fort Tarkos tout à la fois se moque de l'impossibilité logique qui tiraille les discours sur l'après d'un avant, dresse des listes de noms d'artistes comme autant de signes de la modernité et sert une autoproclamation qui fait acte et date: «Je suis l'avantgarde en $1997 »$.

Stéphane Inkel rapproche pour sa part les essais sur la littérature de Pierre Bergounioux et certains de ses récits, tout en continuant de les considérer comme deux ensembles distincts. Et la distinction est en effet fructueuse, car les essais s'éclairent au miroir des récits, et vice versa. Stéphane Inkel montre notamment que, si les références se répondent d'un régime discursif à l'autre, leurs modalités et leurs fonctions diffèrent. Dans ces jeux de décalage, il est dès lors possible de faire émerger "quelque chose comme la résistance du texte bergounien à sa propre théorie». Alors que Stéphane Inkel souligne l'influence directe d'Hegel sur la conception de l'histoire de la littérature de Bergounioux, Nathalie Roy travaille à faire apparaître les liens qui unissent souterrainement la théorie de la littérature de Pascal Quignard à celle des premiers Romantiques allemands. S'attachant à découvrir les déterminations modernes d'une pensée qui s'est elle-même plus volontiers revendiquée de l'héritage classique, examinant les notions de connaissance littéraire, d'extase et d'irreprésentabilité qui hantent son discours, Nathalie Roy explore les commentaires sur la littérature de Quignard, commentaires aussi essentiels qu'abondants puisqu'ils prennent la forme aussi bien de nombreux « petits traités » que d'articles, de conférences ou d'entretiens. 
Les essais de Richard Millet, sur lesquels MartineEmmanuelle Lapointe s'est penchée, se font moins spéculatifs et plus pamphlétaires. C'est d'ailleurs une gradation, un engrenage même, qu'elle fait apparaître dans la série d'essais et d'entretiens publiés par l'auteur entre 2005 et 2008. Elle montre la contradiction qui se noue au fil de ces publications combatives et qui ne cessent pourtant de rêver à une figure de l'écrivain comme clerc, intemporellement situé au-dessus de la mêlée. Le discours sur la littérature est bien cet écran où l'écrivain projette des images de l'écrivain et, dans le même temps, met en scène sa propre figure. Pierre Michon le sait, lui qui ne cesse de jouer avec les masques dans ses entretiens. Virginie Harvey reprend le parallèle cocasse établi par Michon entre l'interview et les séances de spiritisme d'Hugo et en tire une à une les conséquences fort peu frivoles: il permet à Michon de suggérer tout à la fois que l'écrivain est cette sibylle inquiétante et ridicule dont on sollicite constamment les oracles et ce pauvre et obstiné intervieweur de la parole des morts. L'article de Michel Biron, qui clôt ce dossier, saisit à nouveau l'exemple de Michon, mais travaille plus largement autour de cette hypothèse, qui permettrait de saisir une évolution générale du discours sur la littérature des écrivains depuis quelques décennies: s'ils sont globalement moins enclins à publier des manifestes, s'ils endossent moins facilement l'habit $\mathrm{du}$ théoricien de la littérature, ils se font par contre très volontiers les commentateurs de leur condition littéraire et de leur pratique (définie comme "série d'activités bien concrètes, par opposition à l'idée de "règles" ou de "principes" »). Ce déplacement, explique Michel Biron, est le signe d'un changement de paradigme: la figure flaubertienne, qui incarnait l'autonomie de la littérature, a perdu de son 
hégémonie et laisse place à des figures plus représentatives de l'hétéronomie de la littérature.

Les articles réunis dans ce dossier ont d'abord été présentés sous forme de communications lors d'un colloque tenu les 4 et 5 juin 2009 au Département d'études littéraires de l'Université du Québec à Montréal. Cet événement a bénéficié du soutien financier de l'Équipe de recherche sur l'imaginaire contemporain, du Centre de recherche Figura sur le texte et l'imaginaire et du Fonds québécois de recherche sur la société et culture. Nous voudrions aussi remercier Marie-Pascale Huglo, Sylvano Santini et René Audet, qui ont présidé les séances de ce colloque, Julie Parent (www.lestudiocalypso.com), qui en a conçu l'affiche, ainsi que Laurence Côté-Fournier, qui a participé à la révision linguistique et typographique de l'ensemble des articles.

\section{Bibliographie}

GenETTE, Gérard. (1987), Seuils, Paris, Seuil, coll. « Poétique ».

JENNY, Laurent (2002). La Fin de l'intériorité. Théorie de l'expression et invention esthétique dans les avant-gardes françaises (18851935), Paris, PUF, coll. « Perspectives littéraires ».

THIBAUDET, Albert. (2007 [1936]), Histoire de la littérature française, Paris, CNRS Éditions.

VIART Dominique et Bruno VERCIER. (2008 [2005]), La Littérature française au présent. Héritages, modernités, mutations, Paris, Bordas. 